\title{
Neutron star structure in a quark model with excluded volume correction
}

\author{
R. M. Aguirre and A. L. De Paoli \\ Departamento de Física, Facultad de Ciencias Exactas, Universidad Nacional de La Plata, \\ C. C. 67 (1900) La Plata, Argentina
}

(Received 13 November 2002; revised manuscript received 7 July 2003; published 21 November 2003)

\begin{abstract}
We study the effects of the finite size of baryons on the equation of state of homogeneous hadronic matter. The finite extension of hadrons is introduced in order to improve the performance of field theoretical models at very high densities. We simulate the in-medium averaged baryon-baryon strong repulsion at very short distances by introducing a van der Waals-like normalization of the fields. This is done in the framework of the quark meson coupling model that allows to take care of the quark structure of baryons. Since within this model the confinement volume evolves with the fields configuration, the treatment is not equivalent to a simple hard-core potential. We investigate the phase transition to quark matter and the structure of neutron stars. We have found significant corrections at high densities.
\end{abstract}

DOI: 10.1103/PhysRevC.68.055804

PACS number(s): 26.60.+c, 12.39.Ba, 13.75.Ev, 21.30.Fe

\section{INTRODUCTION}

Investigation of hadronic matter at extreme conditions of density and temperature is a current issue of research, since the study of its properties will eventually shed some light over the recovering of QCD symmetries [1,2]. The phase diagram of hadronic matter is expected to be very complex, exhibiting exotic phases such as superfluidity, meson condensates, dibaryon condensate, etc. The gradual emergence of quark droplets would finally lead to a transition to deconfined quark matter. Each of these phenomena affect the equation of state (EOS) and could have macroscopic manifestations, for example, in the structure of stars.

In most hadronic matter studies baryons are assumed to be pointlike. This can be justified because at densities below the nuclear matter saturation density the finite volume effects are expected to be small. However, the spatial extension of baryons was recognized as an essential point in the study of the collective phenomena at very high densities [3].

It is worth mentioning that there are only a few field theoretical models which consistently include the baryonic spatial extension. The most commonly used are the Skyrme and baglike models. In the first case the inclusion of finite baryon density effects is not straightforward due to the topological character of its solutions [4]. On the other hand, further refinements of the original MIT bag model allow one to deal with medium effects on the hadron structure [5-9]. Within this scheme there were recent efforts to include the repulsion between overlapping bags, by means of effective shortranged quark-quark correlations [7].

The authors attempted to take into account finite volume correction previously, using a van der Waals-like method to study nuclear matter with $\Lambda$ hyperons [10]. The total volume appearing in the thermodynamical quantities was replaced by the available volume, in accordance with related investigations [11-15].

In the present work we generalize this approach to study the properties of hadronic matter including the octet of low lying baryons, and to check out their influence on the transition to quark matter. The resulting EOS is applied to study the composition of neutron stars. Following our previous study [10], we introduce these corrections at the level of normalization of the baryon fields in the quark meson coupling (QMC) model $[5,6]$. The motivation for such a procedure is to parametrize in compact form the strong baryonbaryon repulsion at very short distances. We focus on the high density regime, thus it is justified to consider the statistical average of the interaction instead of looking at its details.

In the QMC model the size of the confining volume has its own dynamical evolution, which takes into account the baryonic density and the fields configuration, and it is obtained in a self-consistent calculation. Thus it can somehow be regarded as an effective degree of freedom. The bag radius changes smoothly with the medium properties, and in this sense the finite volume corrections introduced here cannot be considered as a hard-core interaction.

In the following section we give a resume of the QMC model and we introduce the excluded volume correlations. In Sec. III we describe neutron star matter and the phase transition to quark matter. Numerical results and discussions are given in Sec. IV, and conclusions are drawn in Sec. V.

\section{THE QUARK MESON COUPLING MODEL}

Relativistic hadron field theories provide a good description of nuclear matter near the saturation density, and of finite nuclei, too. For this purpose only a small number of free parameters is required. Mean field approximation (MFA) is suited to the aim of these theories, since the treatment of matter at medium and high densities do not require the detailed structure of the interactions. Within this framework the QMC model [5,6] can be viewed as an extension of the quantum hadro-dynamics (QHD) models [16,17].

In the QMC model baryons are represented as nonoverlapping spherical bags containing three valence quarks; the bag radius changes dynamically with the medium density. Baryons interact by the exchange of $\sigma, \omega$ and $\rho$ mesons coupled directly to the confined quarks. It has been found that these extra degrees of freedom, provided by the internal 
structure of the baryon, lead to quite acceptable values of the nuclear matter compressibility at saturation. Despite the explicit quark fields in the QMC model, hadronic thermodynamical properties are evaluated in such a way that baryons are handled as point-like particles with an effective mass $M_{B}^{*}$ which depends on the $\sigma$ field.

In the MFA the Dirac equation for a quark of flavor $q$ ( $q$ $=u, d, s)$, current mass $m_{q}$, and third isospin component $I_{3}^{q}$ is given by

$$
\left(i \gamma^{\mu} \partial_{\mu}-g_{\omega}^{q} \gamma^{0} \omega_{0}-g_{\rho}^{q} I_{3}^{q} \gamma^{0} b_{0}-m_{q}^{*}\right) \Psi^{q}=0 .
$$

In this equation all meson fields have been replaced by their mean field values. Mesons couple linearly only to nonstrange quarks, i.e., $g_{\sigma}^{s}=g_{\omega}^{s}=g_{\rho}^{s}=0$. Therefore the parameters $m_{q}^{*}$ are given by

$$
\begin{gathered}
m_{u, d}^{*}=m_{u, d}-g_{\sigma}^{u, d} \sigma, \\
m_{s}^{*}=m_{s} .
\end{gathered}
$$

For a spherically symmetric bag of radius $R_{b}$ representing a baryon of class $b$, the normalized quark wave function $\Psi_{b}^{q}(r, t)$ is given by

$$
\Psi_{b}^{q}(r, t)=\mathcal{N}_{b}^{-1 / 2} \frac{e^{-i \varepsilon_{q b} t}}{\sqrt{4 \pi}}\left(\begin{array}{c}
j_{0}\left(x_{q b} r / R_{b}\right) \\
i \beta_{q b} \vec{\sigma} \cdot \hat{r} j_{1}\left(x_{q b} r / R_{b}\right)
\end{array}\right) \chi^{q},
$$

where $\chi^{q}$ is the quark spinor and

$$
\begin{gathered}
\varepsilon_{q b}=\frac{\Omega_{q b}}{R_{b}}+g_{\omega}^{q} \omega_{0}+g_{\rho}^{q} I_{3}^{q} b_{0}, \\
\mathcal{N}_{b}=R_{b}^{3}\left[2 \Omega_{q b}\left(\Omega_{q b}-1\right)+R_{b} m_{q}^{*}\right] \frac{j_{0}^{2}\left(x_{q b}\right)}{x_{q b}^{2}}, \\
\beta_{q b}=\left[\frac{\Omega_{q b}-R_{b} m_{q}^{*}}{\Omega_{q b}+R_{b} m_{q}^{*}}\right]^{1 / 2},
\end{gathered}
$$

with $\Omega_{q b}=\left[x_{q b}^{2}+\left(R_{b} m_{q}^{*}\right)^{2}\right]^{1 / 2}$. The eigenvalue $x_{q b}$ is solution of the equation

$$
j_{0}\left(x_{q b}\right)=\beta_{q} j_{1}\left(x_{q b}\right),
$$

which arises from the boundary condition at the bag surface.

In this model the ground state bag energy is identified with the baryon mass $M_{b}^{*}$,

$$
M_{b}^{*}=\frac{\sum_{q} n_{q}^{b} \Omega_{q b}-z_{0 b}}{R_{b}}+\frac{4}{3} \pi B R_{b}^{3},
$$

where $n_{q}^{b}$ is the number of quarks of flavor $q$ inside the bag. The bag constant $B$ represents the difference of energy per unit volume between the vacuum with and without broken QCD symmetry. It is numerically adjusted to get definite values for the proton bag radius. The zeropoint motion parameters $z_{0 b}$ are fixed to reproduce the baryon spectrum at zero density.
Equation (2.8) shows that the baryon effective mass is a function of the bag radius $R_{b}$. In the original MIT bag calculations $R_{b}$ is a constant fixed at zero baryonic density, but in the QMC it is a variable dynamically adjusted to reach the equilibrium of the bag in the dense hadronic medium. We use the equilibrium condition proposed in Ref. [10], which results by imposing a vanishing net flux of the energymomentum tensor through the surface of the bag immersed in the dense hadronic medium. This gives

$$
-\frac{1}{4 \pi R_{b}^{2}}\left(\frac{\partial M_{b}^{*}}{\partial R_{b}}\right)_{\sigma, x}=\frac{1}{3 \pi^{2} \xi} \sum_{b^{\prime}} \int_{0}^{k_{b^{\prime}}} \frac{d k k^{4}}{\sqrt{M_{b^{\prime}}^{* 2}+k^{2}}}
$$

where $\xi=1$. The interested reader can find the detailed derivation of this relation in Ref. [10].

This result reflects the balance of the internal pressure of the bag with the baryonic contribution to the total external pressure, represented by the left-hand and right-hand sides of Equation (2.9), respectively.

The factor $\xi$ will be redefined below when excluded volume effects will be considered.

It must be noted that Eq. (2.9) differs from the standard QMC condition [6], i.e.,

$$
\left(\frac{\partial M_{b}^{*}}{\partial R_{b}}\right)_{\sigma}=0
$$

Both prescriptions coincide only in the case of vanishing density. Equation (2.9) could be more appropriate in dealing with finite density calculations, nevertheless there remains to elucidate the problem of overlapping bags as the density grows. Therefore it becomes necessary to introduce some further considerations into the formalism to handle this feature.

Once $M_{b}^{*}$ has been defined microscopically, the hadronic thermodynamics in the QMC model resembles that of the quantum hadrodynamics. In the MFA for homogeneous infinite static matter all meson fields are replaced by their averaged values, i.e.,

$$
\begin{gathered}
\sigma=\sigma_{0}=-\frac{1}{m_{\sigma}^{2}} \sum_{b} \frac{d M_{b}^{*}}{d \sigma} n_{s}^{b}, \\
\omega_{\mu}=\omega_{0} \delta_{\mu 0}=\frac{1}{m_{\omega}^{2}} \sum_{B} g_{\omega}^{b} n^{b} \delta_{\mu 0}, \\
b_{\mu}^{a}=b_{0} \delta_{\mu 0} \delta_{a 3}=\frac{1}{m_{\rho}^{2}} \sum_{b} g_{\rho}^{b} I_{3}^{b} n^{b} \delta_{\mu 0} \delta_{a 3},
\end{gathered}
$$

where $a=1,2,3$ runs over all isospin directions and $I_{3}^{b}$ is the third isospin component of baryon $b$. In our calculations we have used the values $m_{\sigma}=550 \mathrm{MeV}, m_{\omega}$ $=783 \mathrm{MeV}$, and $m_{\rho}=770 \mathrm{MeV}$ for the meson masses.

The dispersion relation for the $b$ baryon is 


$$
k_{0}^{b}=\sqrt{M_{b}^{* 2}+\vec{k}^{2}} \pm g_{\omega}^{b} \omega_{0} \pm g_{\rho}^{b} I_{3}^{b} b_{0},
$$

for particle $(+)$ and antiparticle $(-)$ solutions. Within the MFA at zero temperature only the particle solutions contribute.

The scalar $\left(n_{s}^{b}\right)$ and baryonic $\left(n^{b}\right)$ densities are defined with respect to the ground state of the hadronic matter $\mid$ g.s. $\rangle$ composed of baryons filling the Fermi sea up to the state with momentum $k_{b}$ :

$$
\begin{gathered}
n_{s}^{b}=\left\langle\text { g.s. }\left|\Psi^{b} \Psi^{b}\right| \text { g.s. }\right\rangle=\vartheta \frac{1}{\pi^{2}} M_{b}^{*} \int_{0}^{k_{b}} d k \frac{k^{2}}{\sqrt{M_{b}^{* 2}+k^{2}}}, \\
n^{b}=\left\langle\text { g.s. }\left|\Psi^{\dagger b} \Psi^{b}\right| \text { g.s. }\right\rangle=\vartheta \frac{k_{b}^{3}}{3 \pi^{2}} .
\end{gathered}
$$

In Eqs. (2.14) and (2.15) the factor $\vartheta$ is included for future use and it takes the value $\vartheta=1$ for pointlike baryons.

In the following section we describe hadronic matter in $\beta$ equilibrium as electrically neutral, therefore we also consider leptons treated as free Dirac particles. The leptonic density $n_{l}$ is related to the Fermi momentum $k_{l}$ by

$$
n^{l}=\left\langle\text { g.s. }\left|\Psi^{\dagger l} \Psi^{l}\right| \text { g.s. }\right\rangle=\frac{k_{l}^{3}}{3 \pi^{2}} .
$$

Given a distribution of baryonic species, we can calculate the total energy density $\epsilon_{H}$ and pressure $P_{0}$ of hadronic matter for pointlike baryons,

$$
\begin{gathered}
\epsilon_{H}=\frac{1}{2} m_{\sigma}^{2} \sigma_{0}^{2}+\frac{1}{2} m_{\omega}^{2} \omega_{0}^{2}+\frac{1}{2} m_{\rho}^{2} b_{0}^{2}+\frac{\vartheta}{\pi^{2}} \sum_{b} \int_{0}^{k_{b}} d k k^{2} \sqrt{M_{b}^{* 2}+k^{2}} \\
+\frac{1}{\pi^{2}} \sum_{l} \int_{0}^{k_{l}} d k k^{2} \sqrt{m_{l}^{2}+k^{2}} \\
P_{0}=\sum_{b} \mu_{0}^{b} n^{b}+\sum_{l} \mu^{l} n^{l}-\epsilon_{H},
\end{gathered}
$$

where $\mu_{0}^{b}=k_{0}^{b}\left(k_{b}\right)$ [see Eq. (2.13)] and $\mu^{l}$ are the chemical potentials for point-like baryons and leptons, respectively.

The QMC model has been widely used to describe the dense hadronic matter; it is based to a great extent on the assumption of nonoverlapping bags. Therefore the breakdown of this hypothesis signals the limit of applicability of the model. Using this criterion an upper density limit around three times the saturation density of symmetric nuclear matter has been found [7]. For densities beyond this value the quark-quark interactions through the confinement region should be introduced, so the naive bag picture is not sufficient to describe the physical situation in this case. This fact is taken into account in the literature using different approaches. For example in Ref. [7] the quark-quark correlations between bags are introduced in the overlapping region. A different approach is given in Ref. [8], where the effects of quark-quark correlations are assumed to be represented by the exponential dependence of the bag constant $B$ on the $\sigma$ meson.
We propose an alternative viewpoint that preserves the scheme of nonoverlapping bags, and that intends to take care of the strong repulsive component of the baryon-baryon interaction that appears as a consequence of the internal structure of the particles. This short-range repulsion can be treated in a simplified model where baryons are described using a dynamics of extended objects. Therefore the fraction of available space is reduced as compared to the case of pointlike particles. A similar approach has been applied to study the phase transition of nuclear matter to the quark-gluon plasma [11,12] and in heavy-ion collisions [13,14].

Since finite size baryons are assumed as nonoverlapping, their motion must be restricted to the available space $V^{\prime}$ defined as $[11,12]$

$$
V^{\prime}=V-\sum_{b} N^{b} v_{b}
$$

with $N^{b}$ the total number of baryons of class $b$ inside the volume $V$ and $v_{b}$ the effective volume per baryon of this class. Hence we conjecture that one can renormalize the particle (antiparticle) wave function replacing $V$ with $V^{\prime}$, and thus the effective baryon fields $\Psi$ can be written as

$$
\begin{aligned}
\Psi^{b}(x)= & \left(V^{\prime}\right)^{-1 / 2} \sum_{\vec{k}, s}\left[a^{b}(\vec{k}, s) u^{b}(\vec{k}, s) e^{-i k^{\mu} x_{\mu}}\right. \\
& \left.+b^{b^{\dagger}}(\vec{k}, s) v^{b}(\vec{k}, s) e^{i k^{\mu} x_{\mu}}\right]
\end{aligned}
$$

in terms of the Fock space operators $a$ and $b$ for particle and antiparticle, respectively. In this way the finite size of the baryons is automatically accounted for into the field dynamics.

Equation (2.20) reinforces the fact that, within this model, the inner and outer regions of the bag must be regarded as complementary in order to avoid inconsistencies. On the contrary, if these regions can overlap, the validity of the linear [Eq. (2.7)] and nonlinear [Eq. (2.9)] boundary conditions should be revised and additional degrees of freedom, such as exotic multiquark states, should be included. Therefore our assumption extends to higher densities the quasiparticle picture and it generates further nonlinear baryonic couplings.

For the moment the volumes $v_{b}$ are parameters associated with the trial quantum state of the whole system, and will be determined using a variational principle, as is explained later.

It is interesting to note that for a mixture of different baryons the excluded volume is not exactly the same for all the species [18]. To simplify the discussions, in this paper we neglect these small differences.

The effective volume per baryon $v_{b}$ is proportional to the actual baryon volume, i.e., for spherical volumes of radius $R_{b}$,

$$
v_{b}=\alpha \frac{4 \pi}{3} R_{b}^{3},
$$

and for sharp rigid spheres $\alpha$ is a real number ranging from 4 , in the low density limit, to $3 \sqrt{2} / \pi$, which corresponds to the maximum density allowed for nonoverlapping spheres, in a face centered cubic arrangement. Since we wish to study the high density regime of homogeneous 
isotropic matter, we shall adopt $\alpha=3 \sqrt{2} / \pi$ in all our calculations. Thus $v_{b}=4 \sqrt{2} R_{b}^{3}$, and the limit of validity of the calculations would correspond to densities $n_{\max }$ such that the center of mass of baryons are at a distance greater than $2 R$ apart. This gives $n_{\max }=\sqrt{2} /\left(8 R_{\max }^{3}\right)$, where $R_{\max }$ denotes the biggest radius among all present baryonic classes. As we shall see below, with the implementation of the procedure outlined in Eq. (2.20) this limit is never reached in the range of densities explored in the present calculations.

In order to see how excluded volume corrections appear in our approach, we shall use the renormalized field of Eq. (2.20) to calculate the relationship among the baryonic densities and the Fermi momenta $k_{b}$,

$$
n^{b}=V^{-1} \int_{V} d x^{3}\left\langle\text { g.s. }\left|\Psi^{\dagger b} \Psi^{b}\right| \text { g.s. }\right\rangle=\left(1-\sum_{b^{\prime}} n^{b^{\prime}} v_{b^{\prime}}\right) \frac{k_{b}^{3}}{3 \pi^{2}},
$$

where $\Sigma_{k} \rightarrow V^{\prime} /\left(2 \pi^{3}\right) \int d k^{3}$ has been used.

This result is equivalent to Eq. (2.15) if the factor $\vartheta$ takes the value

$$
\vartheta=1-\sum_{b} n^{b} v_{b}
$$

for finite baryonic effective volumes $v_{b}$. In the limit $v_{b}$ $\rightarrow 0$ one recovers the pointlike expressions.

Equation (2.22) shows that these kinds of short-range correlations couple nonlinearly the baryons among themselves, in a density dependent way. Thus baryons are considered in this scheme as quasiparticles dressed with these corrections.

The density $n_{b}$ for a given baryonic species appears on both sides of Eq. (2.22), and it is possible to solve it exactly for $n_{b}$ in terms of all the Fermi momenta, namely,

$$
n^{b}=\frac{1}{\left(1+\sum_{b^{\prime}} \frac{k_{b^{\prime}}^{3}}{3 \pi^{2}} v_{b^{\prime}}\right)} \frac{k_{b}^{3}}{3 \pi^{2}} .
$$

Since $\vartheta$ depends explicitly on the baryonic densities, the chemical potentials get an extra term, i.e.,

$$
\begin{gathered}
\mu^{b}=\left(\frac{\partial \epsilon_{H}}{\partial n_{b}}\right)_{\substack{n_{b^{\prime}} \\
b^{\prime} \neq b}}=\mu_{0}^{b}+\Delta \mu^{b}, \\
\Delta \mu^{b}=\frac{v_{b}}{3 \pi^{2}} \sum_{b^{\prime}} \int_{0}^{k_{b^{\prime}}} \frac{d k k^{4}}{\sqrt{M_{b^{\prime}}^{* 2}+k^{2}}},
\end{gathered}
$$

where the energy density $\epsilon_{H}$ is given by Eq. (2.17) with $\vartheta$ defined as in Eq. (2.23).

The total pressure acquires an additional term $\Delta P$ as compared to the pressure of pointlike baryons $P_{0}$ in Eq. (2.18),

$$
P_{H}=P_{0}+\Delta P=P_{0}+\sum_{b} n^{b} \Delta \mu^{b} .
$$

We proceed to give a physical interpretation of the volumes $v_{b}$ of the baryons, or equivalently their radii $R_{b}$. They are considered within this approach as variational parameters of the trial quantum state of the system. Their equilibrium values, at zero temperature, must be determined by minimizing the total energy of the whole system, in agreement with Refs. [11,12]. This procedure defines unambiguously the equilibrium radii of the bags, which depend on the baryonic density.

The new equilibrium condition for the bag consistent with the excluded volume corrections introduced in Eq. (2.20) has the same form as that given in Eq. (2.9), but now $\xi$ $=n_{s}^{b} /\left(\alpha n^{b}\right)$.

We shall see in the following sections that as the density grows a state of deconfined quarks becomes more favorable than a system of quarks confined within baryons. Because of this, the concept of excluded volume is meaningless when the baryonic phase has dissappeared completely.

In our approach only the baryonic states receive an explicit correction due to short-range correlations, by the normalization of the fields $\Psi^{b}$. It must be stressed that the meson mean field values are completely determined from the baryonic sources that already include finite size corrections in $\vartheta$. Leptons do not couple to strong interactions, and in this sense they are taken as pointlike particles.

To summarize, in the QMC model extended to high densities the hadronic matter properties are determined applying the set of equations (2.7) to Eq. (2.17), together with Eqs. (2.25)-(2.27), for a fixed total baryonic density $n$, using the value of $\vartheta$ given in Eq. (2.23).

\section{QUARK MATTER PHASE TRANSITION AND THE STRUCTURE OF NEUTRON STARS}

Neutron star matter is electrically neutral and it has reached equilibrium against $\beta$ decay. The relative abundance of the different baryonic species are determined by these conditions. The structure of neutron stars with hyperon contributions has been widely studied, for recent investigations see, for example, Refs. [8,19], and references therein. In particular, in Ref. [8] an extension of the QMC model is used, which takes into account the density dependence of the bag constant $B$ and includes strange mesons.

In the present work we consider the nucleon duplet $(n, p)$, the $\Lambda$ hyperon, the $\Sigma$ triplet, the $\Xi$ duplet, and two lepton species, electron and muon.

In hadronic matter at chemical equilibrium the following relationships are fulfilled for the baryonic and leptonic chemical potentials

$$
\begin{gathered}
\left.\mu^{b}=\mu^{n}+Q_{b} \mu^{e} \text { (if baryon class } b \text { is present }\right), \\
\mu^{e}=\mu^{\mu} \text { (if muons are present), }
\end{gathered}
$$

with $Q_{b}$ the electric charge in units of the positron charge for the class $b$ of baryons, $\mu^{b}$ given by Eq. (2.25), and $\mu^{l}=k_{0}^{l}\left(k_{l}\right)=\sqrt{m_{l}^{2}+k_{l}^{2}}$.

Mean field equations are solved for fixed total baryonic density $n$ and zero total electric charge density,

$$
n=\sum_{b} n^{b},
$$




$$
0=\sum_{b} Q^{b} n^{b}-\sum_{l} n^{l}
$$

where the sums run over the baryonic octet and over the two lepton species, respectively.

Once Eqs. (2.7)-(2.9) have been solved, from Eqs. (2.10)-(2.12) we determine the meson fields $\left(\sigma_{0}, \omega_{0}, b_{0}\right)$ and together with Eqs. (2.15) and (3.1)-(3.4) we get the baryonic and leptonic densities $\left(n^{b}, n^{l}\right)$, all the Fermi momenta $\left(k_{b}, k_{l}\right)$, and chemical potentials $\left(\mu^{b}, \mu^{l}\right)$.

As the baryonic density increases a phase transition from hadronic to quark matter, made up with deconfined quarks, can be reached. Therefore the previous set of equations must be modified to satisfy the new conditions of $\beta$ equilibrium in the transition region. Since the baryonic number and the electric charge neutrality are always conserved, a smooth crossover between hadronic and quark matter must be expected because there are two conserved charges. In fact, in this coexistence region of mixed hadron and quark phases the total baryonic density and the total (zero) electric charge are shared between these two phases.

To determine the composition of the transition region we suppose that quarks can exist either in the confined phase (inside baryons) or as deconfined particles. As a first approximation we suppose that the deconfined phase contains free quarks and leptons, and nonperturbative gluon effects are represented by the bag constant $B$.

Under equilibrium conditions, the net flux of quarks through the bag surface is zero, and hence the two phases coexist with the only constraint of globally conserved electric and baryonic charges.

We adopt the treatment of Ref. [20] as it is appropriate to describe phase transitions with more than one conserved charge. Thus, in the coexistence phase, the conservation equations (3.3) and (3.4) are generalized to [20]

$$
\begin{gathered}
n=(1-\chi) n_{H}+\chi n_{Q}, \\
0=(1-\chi) \sum_{b} Q^{b} n^{b}+\chi \sum_{q} Q^{q} n^{q}-\sum_{l} n^{l},
\end{gathered}
$$

where $n_{H}=\Sigma_{b} n^{b}, n_{Q}=\Sigma_{q} n^{q} / 3$ are, respectively, the hadron and quark contribution to the baryon number density; $n^{q}$ $=N_{c} k_{q}^{3} /\left(3 \pi^{2}\right)$ is the number density of quarks for $N_{c}=3$ colors, $k_{q}$ is the Fermi momentum, and $Q^{q}$ is the electric charge in units of the positron charge for quarks of flavor $q$. The quantity $\chi$ is the volume fraction corresponding to the quark matter phase $(0 \leqslant \chi \leqslant 1)$. The $\beta$-equilibrium condition for quarks in the deconfined phase reads $\mu_{d}=\mu_{s}$ $=\mu_{u}+\mu_{e}$, with $\mu_{q}=\sqrt{m_{q}^{2}+k_{q}^{2}}$. In the mixed phase these relations must be supplemented with $\mu_{n}=3 \mu_{d}-\mu_{e}$ and the mechanical equilibrium condition $P_{H}=P_{Q}$, where $P_{H, Q}$ are the pressures in each phase. It must be noted that the case $\chi=0$ (1) in Eq. (3.5) corresponds to the pure hadronic (quark) matter phase instance, the mixed phase is in between.

The energy density in the mixed phase can be similarly expressed as $\epsilon=(1-\chi) \epsilon_{H}+\chi \epsilon_{Q}$. The quantities $\epsilon_{Q}$ and $P_{Q}$ are, respectively, the energy density and pressure for the deconfined phase which contains free quarks and leptons [20],

$$
\epsilon_{Q}=B+\frac{N_{c}}{\pi^{2}} \sum_{q} \int_{0}^{k_{q}} d k k^{2} \sqrt{m_{q}^{2}+k^{2}}+\frac{1}{\pi^{2}} \sum_{l} \int_{0}^{k_{l}} d k k^{2} \sqrt{m_{l}^{2}+k^{2}}
$$

$$
P_{Q}=\sum_{q} \mu_{q} n^{q}+\sum_{l} \mu_{l} n^{l}-\epsilon_{Q} .
$$

Nonperturbative effects in $\epsilon_{Q}$ arising from the gluons are represented by the bag constant $B$ [20].

Of course, when the pure quark matter phase is reached $(\chi=1)$, the conditions of chemical equilibrium apply among deconfined quarks, since this means that baryons have solved completely.

The EOS emerging from this calculation can be used to evaluate the properties of neutron stars. The stellar radius $R$ and mass $M$ are obtained by solving the TolmanOppenheimer-Volkoff relativistic equations for a spherically symmetric (nonrotating) neutron star:

$$
\begin{gathered}
\frac{d P}{d r}=-\left(G / c^{2}\right) \frac{[\epsilon(r)+P(r)]\left[m(r)+4 \pi r^{3} P(r) / c^{2}\right]}{r^{2}\left[1-2\left(G / c^{2}\right) m(r) / r\right]}, \\
m(r)=\int_{0}^{r} 4 \pi r^{\prime 2}\left[\epsilon\left(r^{\prime}\right) / c^{2}\right] d r^{\prime} .
\end{gathered}
$$

Starting from a given value $\epsilon_{c}$ for the central energy density, these equations are integrated outward until a radius $R\left(\epsilon_{c}\right)$ is reached for which the pressure $P$ is zero, and $M$ $=m(R)$ is defined.

To determine more accurately the radius of the star, it becomes necessary to use an appropriate EOS for low densities. We have selected the EOS given in Ref. [21] for baryonic densities below $0.1 n_{0}$. It is worth mentioning that another possibility is to choose the EOS given in Ref. [22], which proves to be very similar to the former [21].

The moment of inertia $I$ for a slowly rotating star can be obtained using $[21,23]$

$$
I=\frac{8 \pi}{3} \int_{0}^{R} r^{4} e^{-\nu(r) / 2} \frac{[\epsilon(r)+P(r)] / c^{2}}{\sqrt{1-2\left(G / c^{2}\right) m(r) / r}} \frac{\bar{\omega}(r)}{\Omega} d r,
$$

where $\Omega$ is the uniform angular velocity of the star as seen by a distant inertial observer, $\Omega \ll(c / R) \sqrt{\left(G / c^{2}\right) M / R}$. The radial function $\nu(r)$ is solution of the differential equation

$$
\frac{d \nu}{d r}=2\left(G / c^{2}\right) \frac{\left[m(r)+4 \pi r^{3} P(r) / c^{2}\right]}{r^{2}\left[1-2\left(G / c^{2}\right) m(r) / r\right]},
$$

with the boundary condition $\nu(R)=-\ln \left[1-2\left(G / c^{2}\right) M / R\right]$. The relative angular velocity $\bar{\omega}(r)$ measured with respect to the local dragged inertial frame is the solution of

$$
\frac{d}{d r}\left[r^{4} j(r) \frac{d \omega(r)}{d r}\right]+4 r^{3} \bar{\omega}(r) \frac{d j}{d r}=0,
$$

with $j(r)=\sqrt{1-2\left(G / c^{2}\right) m(r) / r} \exp [-\nu(r) / 2]$ inside the star. Since $j(r)=1$ for $r \geqslant R, \bar{\omega}(r)$ has the form $\bar{\omega}(r)=\Omega[1$ $\left.-2\left(G / c^{2}\right) I / r^{3}\right]$ outside. 
TABLE I. The quark-meson couplings $g_{\sigma, \omega, \rho}^{u, d}$ for each of the three values of the bag constant $B$ used in our calculations, and for each of the approaches with excluded volume correction (CC) and without it (NC). The following two columns show the lower $\left(n_{c l}\right)$ and upper $\left(n_{c u}\right)$ densities of the mixed hadron-quark phase, and in the last column the limiting density $n_{\max }$ for the NC cases is displayed.

\begin{tabular}{lcccccccc}
\hline \hline$B^{1 / 4}(\mathrm{MeV})$ & $R_{p}(\mathrm{fm})$ & Case & $g_{\sigma}^{u, d}$ & $g_{\omega}^{u, d}$ & $g_{\rho}^{u, d}$ & $n_{c l} / n_{0}$ & $n_{c u} / n_{0}$ & $n_{\max } / n_{0}$ \\
\hline 169.93 & 0.8 & $\mathrm{NC}$ & 5.747 & 2.756 & 8.668 & 1.20 & & 3.40 \\
& & $\mathrm{CC}$ & 4.678 & 1.701 & 7.600 & 1.06 & 3.98 & \\
187.83 & 0.7 & $\mathrm{NC}$ & 5.858 & 2.872 & 8.582 & 2.10 & & 6.40 \\
& & $\mathrm{CC}$ & 5.311 & 2.372 & 7.948 & 1.87 & 6.58 & \\
210.85 & \multirow{2}{*}{0.6} & $\mathrm{NC}$ & 5.993 & 3.007 & 8.523 & & & 6.20 \\
& & $\mathrm{CC}$ & 5.702 & 2.747 & 8.151 & 3.91 & 10.17 & \\
\hline \hline
\end{tabular}

A useful quantity in astronomical observations is the surface redshift $z$ given by

$$
z=\left[1-2\left(G / c^{2}\right) M / R\right]^{-1 / 2}-1 .
$$

Numerical values obtained in our approach can be found in Table II.

\section{NUMERICAL RESULTS}

Within the present model the quark masses take the current values $m_{u}=m_{d}=5 \mathrm{MeV}$ and $m_{s}=150 \mathrm{MeV}$. The parameter $B$ is the same for all the baryon bags, and we avoid any speculation about its density dependence. For a given value of $B$ the set of parameters $z_{0 b}$ are adjusted to obtain the experimental baryon masses at zero density.

Since mesons interact directly with quarks, the corresponding meson-baryon couplings are related to the quarkmeson couplings $g_{\phi}^{q}(\phi=\sigma, \omega, \rho, q=u, d)$ in a simple way [6]. Denoting as $g_{\phi}^{b}$ the coupling of the $\phi$ meson to $b$ baryon,

$$
\begin{gathered}
g_{\sigma}^{b}=N_{n s}^{b} g_{\sigma}^{u}, \\
g_{\omega}^{b}=N_{n s}^{b} g_{\omega}^{u}, \\
g_{\rho}^{b}=g_{\rho}^{u},
\end{gathered}
$$

where $N_{n s}^{b}$ is the nonstrange quark number inside the baryon $b$. Thus, for given $g_{\sigma}^{u}, g_{\omega}^{u}$, and $g_{\rho}^{u}$, the full set of baryon-meson couplings can be determined. Their numerical values are obtained by reproducing the symmetric nuclear matter properties at saturation, i.e., baryonic density, binding energy, and symmetry energy,

$$
\begin{gathered}
n_{0}=0.15 \mathrm{fm}^{-3}, \\
E_{b}=(\epsilon / n)_{0}-M c^{2}=-16 \mathrm{MeV}, \\
a_{s}=\frac{1}{2}\left(\frac{\partial^{2}(\epsilon / n)}{\partial t^{2}}\right)_{t=0}=35 \mathrm{MeV},
\end{gathered}
$$

where $t=\left(n_{n}-n_{p}\right) / n$ and $M=938.92 \mathrm{MeV} / c^{2}$ is the averaged free nucleon rest mass.

As expected, the values of the couplings are sensitive to either the inclusion or not of the excluded volume corrections. Both instances are considered in Table I.
For practical applications, we have chosen the values $B^{1 / 4}=169.93,187.83$, and $210.85 \mathrm{MeV}$ that will be denoted, respectively, as (a), (b), and (c) in the following. They yield a proton bag radius $R_{p}=0.8,0.7$, and $0.6 \mathrm{fm}$, respectively (see Table I). The cases with the excluded volume corrections (CC) have been compared with calculations without them (NC). We remark that in the cases labeled NC we take $\xi=\vartheta=1$, and $\Delta \mu^{b}=0$ for all baryons.

The same $B$ is used consistently in the QMC and deconfined quark descriptions.

In all cases we start in the hadronic phase increasing the baryonic density until the coexistence conditions with quark matter are satisfied. The exception here is the case (c)-NC that stays always in the hadronic phase, at least within the range of validity of the nonoverlapping bag assumption.

This phase transition occurs at critical densities $n_{c l}$ which are shown in Table I; it can be seen that $n_{c l}$ increases with $B$. The lowest value $n_{c l} \simeq n_{0}$ corresponds to $B^{1 / 4}=169.93 \mathrm{MeV}$, and therefore we interpret that it is not suitable for a true physical consideration and has been kept only for illustrative purposes.

For the bag parameters $B$ considered here, the nonoverlapping bag hypothesis breaks down in the $\mathrm{NC}$ approach at limiting densities $n_{\max }$, before the pure quark plasma state has taken place. These upper limits are displayed in the last column of Table I. Thus, stars with a pure quark core could not be described within this framework.

On the other hand, in the $\mathrm{CC}$ instance the upper density threshold $n_{c u}$ for the mixed phase is enlarged as $B$ increases. No bag overlapping is produced in the calculations using the $C C$ option for densities $n<n_{c u}$, and for $n>n_{c u}$ excluded volume effects are meaningless because only quark matter without hadrons can be found.

In Fig. 1 we plot the contribution of the hadronic phase $\left(n_{H}\right)$ and the free quark phase $\left(n_{Q}\right)$ to the total baryonic density in the mixed phase, see upper equation of Eqs. (3.5). For the sake of completeness we draw a dotted line corresponding to matter in a pure (either hadronic or quark) phase, all curves above (below) this line represent the contributions $n_{Q}\left(n_{H}\right)$. The case (c)-NC stays in the pure hadronic phase for all ranges of densities studied, and therefore it coincides with the dotted line in this plot. It can be seen that $n_{H}$ lies always below $5.6 n_{0}$, which corresponds to the topmost case [(c)-CC] considered here. 


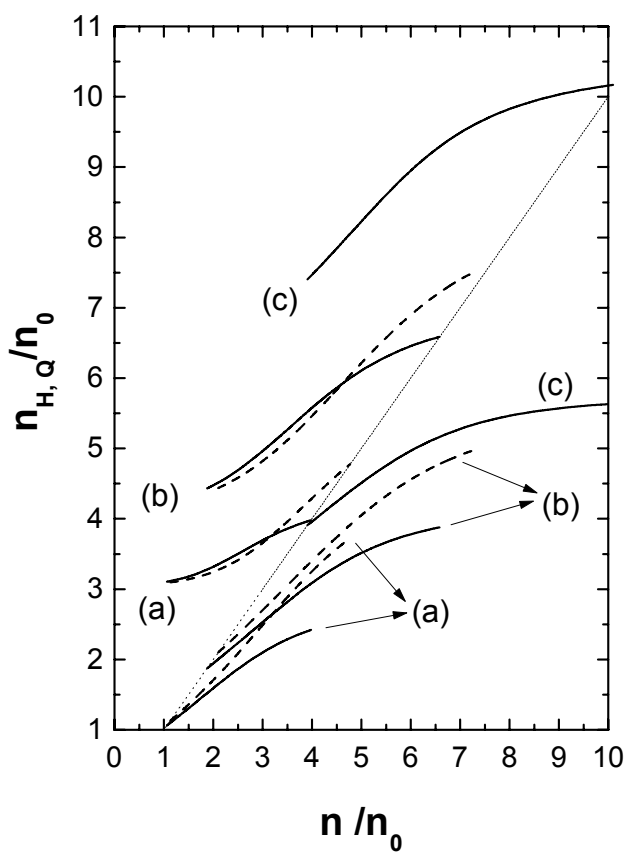

FIG. 1. Hadronic $\left(n_{H}\right)$ and quark $\left(n_{Q}\right)$ contributions to the total baryonic density in the mixed phase. The results with (without) excluded volume correction are represented with solid (dashed) lines. The different bag constant values $B^{1 / 4}=169.9,187.8$ and $210.8 \mathrm{MeV}$ are distinguished with the labels (a), (b), and (c), respectively. For comparison we have also drawn a curve corresponding to a pure phase (dotted line). All curves above (below) this line represent the contributions $n_{Q}\left(n_{H}\right)$. The case (c) NC stays in the pure hadronic phase for all ranges of densities studied, and therefore coincides with the dotted line in this graph. The $\mathrm{CC}$ curves are plotted up to the upper density threshold $n_{c u}$. Numerical values for the density threshold $n_{c l}$ and $n_{c u}$ can be seen in Table I.

In Fig. 2 we show the meson mean field values in the hadronic and mixed phases. Differences between the CC and NC treatments become appreciable for $n>n_{0}$. As can be seen, the $\rho$ meson amplitude has a sudden change of slope at the phase transition due to the change in the isospin composition of the hadronic sector in the neighborhood of $n_{c l}$. In the same figure the factor $\vartheta$ containing the excluded volume correction is displayed as a function of the baryonic density. This factor seems to become almost constant at sufficiently high densities.

The proton bag radius $R_{p}$ and its effective mass $M_{p}^{*}$ can be examined in Fig. 3. A faster radius decrease is observed in the $\mathrm{CC}$ instance, as compared with the respective $\mathrm{NC}$ calculation. This fact explains the absence of overlap and the consequent lengthening of the validity range of the $\mathrm{CC}$ approach. A dropping of about $15-20 \%$ in $R_{p}$ is predicted before the hadronic matter completely disappears; this strong compression of the baryon bags helps the deconfinement mechanism in the CC case. This can be understood because at a given density the pressure and the chemical potential of baryons are slightly greater when the excluded volume correlations are included than in the $\mathrm{NC}$ results. Bearing in mind that at the transition point there is a crossing of the curves representing the pressure in terms of the chemical potential
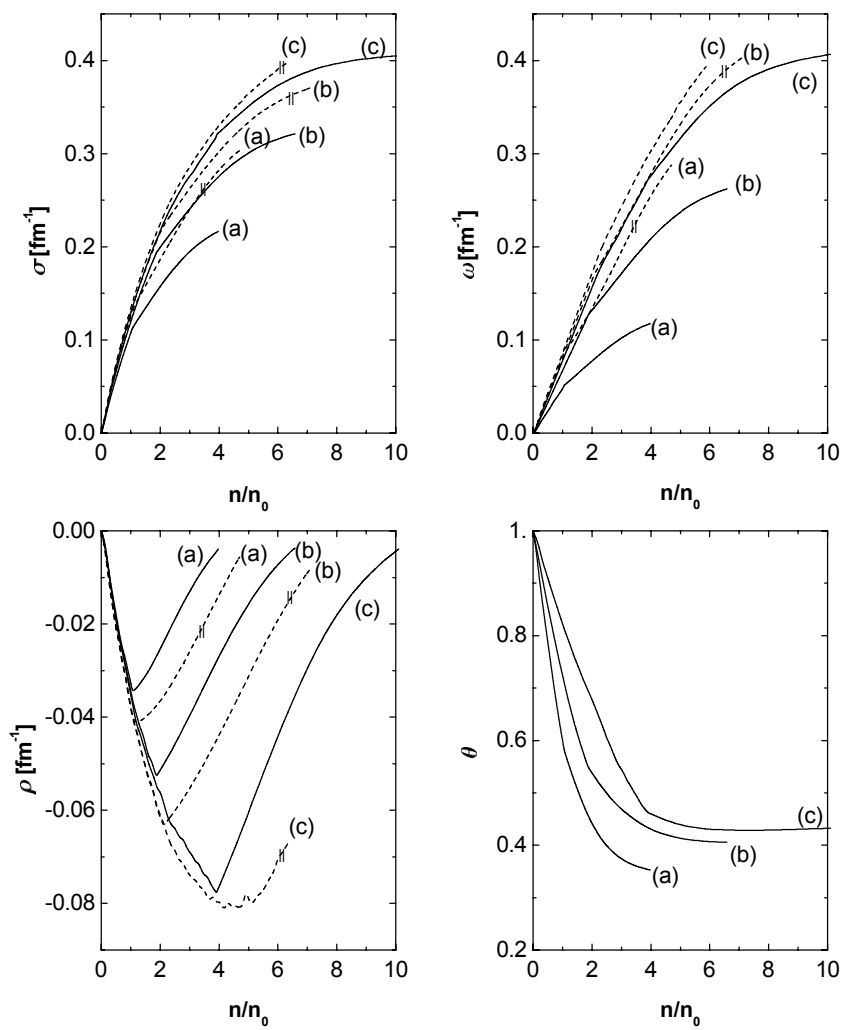

FIG. 2. Meson mean field values $\sigma, \omega$, and $b_{0}$ as functions of the baryonic density relative to the saturation nuclear density $n_{0}$. The line and label conventions are the same as in Fig. 1. In the right lower corner the volume correction factor $\vartheta$ is displayed in terms of the baryonic density. The double vertical bars indicate the limit of the nonoverlap assumption for the $\mathrm{NC}$ cases.

for the hadronic and quark phases, this crossing occurs at lower densities for the $\mathrm{CC}$ than for the $\mathrm{NC}$ case. On the other side, due to the slow decrease of the bag radius in the $\mathrm{NC}$ approach, the overlapping of bags can happen before the phase transition to pure quark matter takes place.

The effective mass $M_{p}^{*}$ exhibits a monotonous decrease as a function of the baryonic density, the rate of variation at low
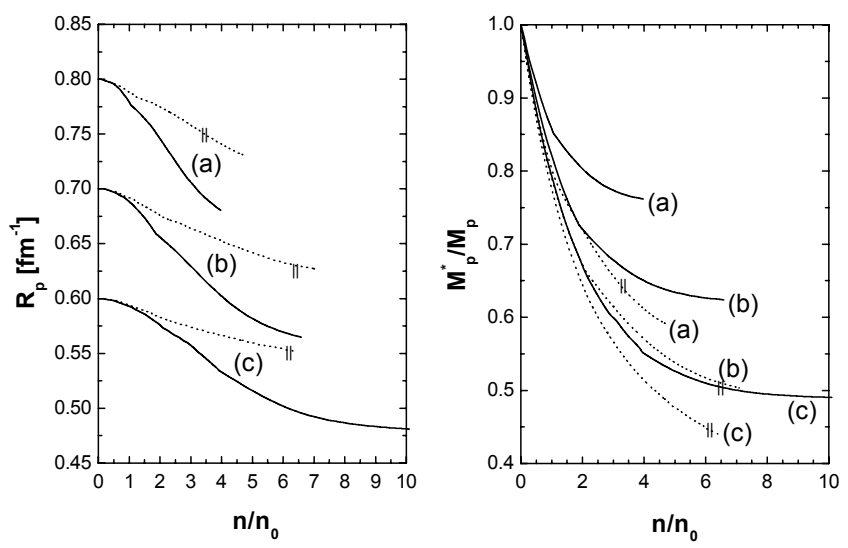

FIG. 3. The in-medium proton bag radius $R_{p}$ and proton mass $M_{p}^{*}$ relative to its vacuum values. The line and label conventions are the same as in Fig. 2. 

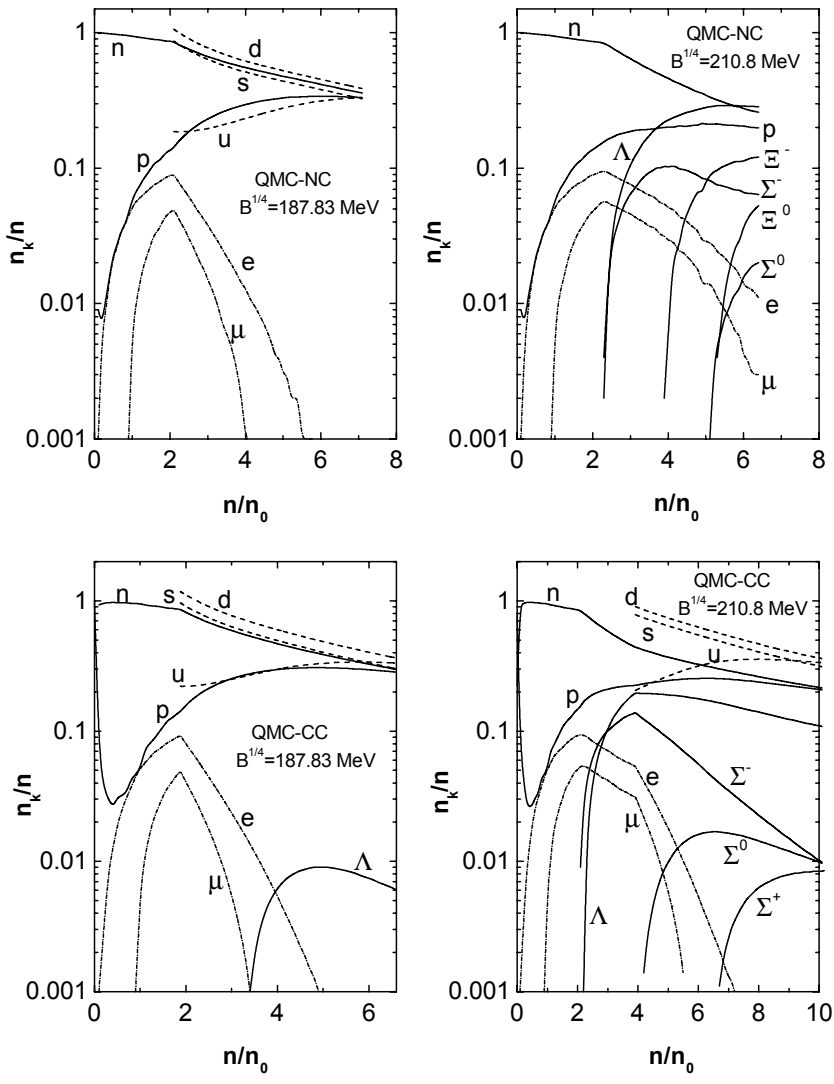

FIG. 4. The baryon and quark composition of the star matter in terms of the baryonic density for the bag constant values $B^{1 / 4}$ $=187.8$ and $210.85 \mathrm{MeV}$ for the $\mathrm{NC}$ and $\mathrm{CC}$ cases. The line convention is explained in each panel.

densities is attenuated by both decreasing $B$ and/or including volume corrections.

The composition of star matter is depicted in Fig. 4 for cases (b) and (c). The onset of the quark phase in the $\mathrm{NC}$ instance suppresses the hyperons that could be present if this transition had not happened. Otherwise in the CC approach new hyperon species appear even in the mixed phase. The pronounced decrease of the lepton abundance in the mixed phase must be noted.

The equation of state for (b) and (c) is represented in Fig. 5. The energy density $\epsilon$ in the mixed phase varies in the range $0.3-1.1 \mathrm{GeV} \mathrm{fm}^{-3}$ for (b)-CC, whereas it ranges between $0.6-2.0 \mathrm{GeV} \mathrm{fm}^{-3}$ for the set (c)-CC. In the CC treatment the pressure shows sudden changes of slope at the extreme points of the mixed phase, which are absent in the $\mathrm{NC}$ case.

The results for neutron star masses and radii based on these equations of state are displayed in Fig. 6. They are given in units of the solar mass $M_{\odot}=1.9889 \times 10^{30} \mathrm{~kg}$.

The masses $\bar{M}$, radii $\bar{R}$, and moments of inertia $\bar{I}$ for the maximum mass star are listed in Table II. We have found that $1.51 \leqslant \bar{M} / M_{\odot} \leqslant 1.88$, a result which is above the experimental lower limit $M / M_{\odot}=1.44$ accepted for binary pulsars.

For these particular cases we have obtained that the radii $\bar{R}$ are rather insensitive to the star internal structure, ranging between $12 \mathrm{~km} \lesssim \bar{R} \lesssim 13 \mathrm{~km}$, being slightly bigger when ex-

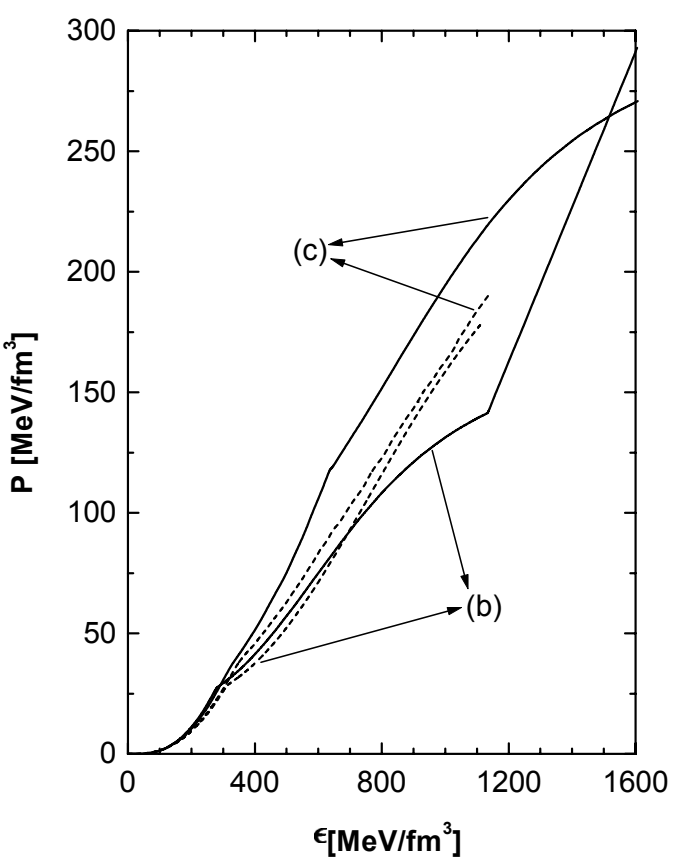

FIG. 5. The pressure $P$ of star matter in terms of the total energy density $\epsilon=E / V$, for the bag constant values $B^{1 / 4}=187.8$ and $210.85 \mathrm{MeV}$, for the NC (dashed lines) and CC (solid lines) cases. For the last instance the pressure has discontinuous derivatives at $n_{c l}$ and $n_{c u}$ (see Table I).

cluded volume corrections are included. Figure 6 also shows that the maxima are reached in a plateau, and they are enhanced in the $\mathrm{CC}$ cases studied here with respect to the $\mathrm{NC}$ ones (Table II). One can also appreciate what happens when excluded volume effects are not considered. In fact, the NC EOS (dashed lines) predicts that for stars approaching the maximum stable mass $\bar{M}$ the baryonic bags at the center are
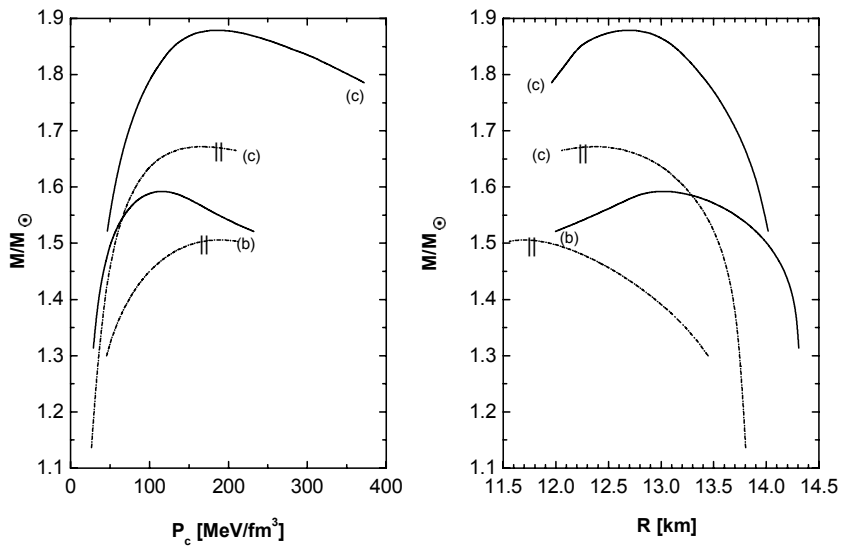

FIG. 6. The gravitational star mass $M / M_{\odot}$ in terms of the central pressure $P_{c}$ (left panel) and $M / M_{\odot}$ in terms of the star radius $R$ (right panel). It can be appreciated that excluded volume corrections enhance both $\bar{M}$ and $\bar{R}$, CC cases (solid lines). Numerical values can be examined in Table II. The double vertical bars have the same meaning as in Fig. 2, indicating that in the NC cases (dashed lines) the bags at the center of the star are close to overlap for masses near the maximum. 
TABLE II. Neutron star properties for each of the bag constants used in our calculations and for each of the approaches: with excluded volume correction (CC) and without it (NC). The star mass $\bar{M}$ relative to the sun mass, the star radius $\bar{R}$, the central baryonic density $\left(n_{c \max }\right)$, the surface redshift $\bar{z}$, and the moment of inertia $\bar{I}$ (for a slowly rotating star), all corresponding to the star with maximum mass.

\begin{tabular}{lcccccc}
\hline \hline$B^{1 / 4}(\mathrm{MeV})$ & Case & $\bar{M} / M_{\odot}$ & $\bar{R}(\mathrm{~km})$ & $n_{c \max } / n_{0}$ & $\bar{z}$ & $\bar{I}\left(10^{30} \mathrm{~kg} \mathrm{~km}^{2}\right)$ \\
\hline 187.83 & $\mathrm{NC}$ & 1.506 & 11.70 & 6.886 & 0.2701 & 137.8 \\
& $\mathrm{CC}$ & 1.592 & 13.02 & 5.148 & 0.2513 & 184.1 \\
210.85 & $\mathrm{NC}$ & 1.672 & 12.38 & 5.800 & 0.2897 & 181.5 \\
& $\mathrm{CC}$ & 1.879 & 12.69 & 5.507 & 0.3330 & 227.1 \\
\hline \hline
\end{tabular}

close to overlap. This is indicated by vertical double bars on the corresponding curves. In particular for the case (b)-NC the bags are overlapping at the center of $\bar{M}$, see Tables I $\left(n_{\max }\right)$ and II $\left(n_{\text {cmax }}\right)$. On the other hand, when CC corrections are included no overlap is present and this is one of the points in favor of the inclusion of the excluded volume correlations.

At this point we can compare our predictions with previous works, for example, Ref. [8] where the QMC model is used to study hyperonic matter and neutron star properties. There the bag constant depends on the scalar mesons fields, and the increase of the radius with density leads to an early overlap of the bags. This feature contrasts with the monotonous decrease of the bag radii we have found. We can also examine the EOS, although the transition to quark matter is not considered in Ref. [8]. The model named QMCI in this reference and the set (b)-NC predict a similar EOS, and for the option (b)-CC we find a slightly stiffer behavior in the pure hadronic matter region due to excluded volume corrections. At higher densities the appearance of the mixed phase softens significantly the EOS, as is shown in Fig. 5. With respect to the maximum neutron star mass and size, the case (b)-NC (Table II) compares fairly well with those given by the model QMCI [8].

The structure of the star, in terms of shells of either pure quark, mixed quark-hadron, or pure hadron matter, depends on the selected value of the bag parameter $B$. This can be seen in Fig. 7 for the CC (b) and (c) cases, where we plot the mass $m(r)$ (left panel) and moment of inertia $I(r)$ (right panel) enclosed within a given $r$, for the maximum star masses (Table II). The mass $m(r)$ is given in Eq. (3.8), and $I(r)$ corresponds to the definition given in Eq. (3.9) with the replacement $R \rightarrow r$ in the upper integration limit. As indicated in this figure, the case (b), CC, predicts that a mixed phase of deconfined quarks and hadrons can be found in a central core with a radius of about $7.48 \mathrm{~km}$, which contains $45 \%$ of $\bar{M}$ and contributes $18 \%$ to the moment of inertia $\bar{I}$ of the star. Meanwhile in the (c)-CC case the hadron-quark mixed phase extends up to $4.31 \mathrm{~km}$ and it encloses only $12 \%$ of $\bar{M}$; its contribution to $\bar{I}$ is small, around $1.5 \%$.

For the sake of completeness, we mention that the option (a)-CC, although not physically reliable, predicts for the star with the maximum mass a structure which includes a pure quark core of $6.55 \mathrm{~km}$. This core is surrounded by a crust of mixed phase located between $6.55 \mathrm{~km}$ and $8.04 \mathrm{~km}$, and the outer shell contains only hadronic matter. The mass of this star is $1.42 M_{\odot}$ with a radius of $9.28 \mathrm{~km}$.

\section{CONCLUSIONS}

We have studied the high density regime of matter in a schematic model of quarks confined into bags, the so called QMC model. One of the main hypotheses of this model is that bags do not overlap, but this situation is reached at relatively low densities of around three times the nuclear matter saturation density in the fixed $B$ treatment. Two different approaches have been given to solve this problem by relaxing the requirement of nonoverlapping bags [7,8]. However these procedures could give rise to formal inconsistencies such as the nonvalidity of the boundary conditions at the bag surface. This point should be clarified before practical applications at extreme densities can be done.

In the present work we intend to retain all the formal aspects of the original QMC, including at the same time the dynamical effects arising at very high densities. We do this by modifying the standard QMC treatment in two aspects, in the first place we consider an alternative condition for the equilibrium of a bag immersed in a dense medium. In the second place we introduce in-medium averaged short-range
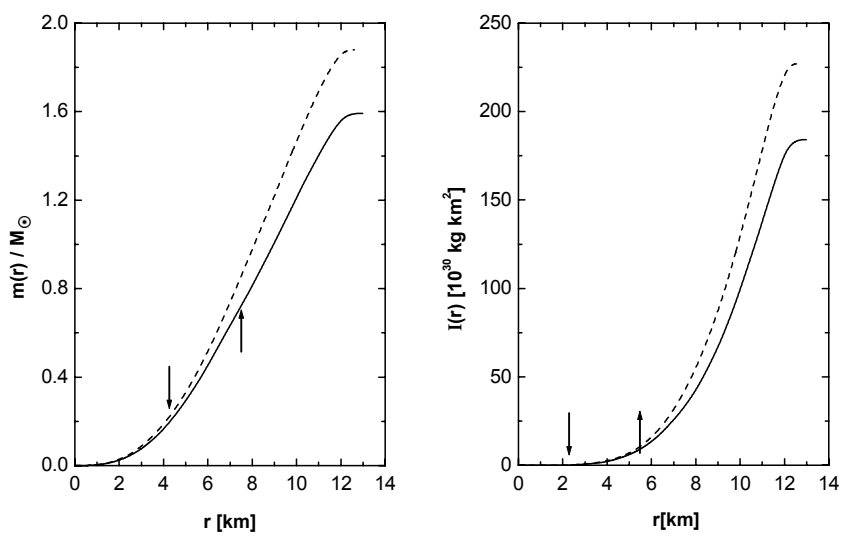

FIG. 7. The star mass (moment of inertia) enclosed within the spherical region of radius $r$ [see Eqs. (3.8) and (3.9)] is shown in the left (right) panel for the sets (b) (solid line) and (c) (dashed line) within the $\mathrm{CC}$ treatment. These results correspond to the maximum star mass $\bar{M}$, as listed in Table II. The mixed phase threshold is marked with a vertical arrow. 
correlations among baryons by using an excluded volume treatment. Thus we obtain a quasiparticle picture of baryons dressed by these strong correlations, which remains valid in all ranges of the present calculations.

We studied the equation of state of hadronic matter and the transition to a deconfined quark phase. Our approach links coherently the nonperturbative QCD effects represented by the bag constant $B$, for both phases of confined and deconfined quarks. The excluded volume correction becomes effective at intermediate densities, contributing to the onset of a quark matter phase. The nonoverlapping bag hypothesis is verified over all ranges of densities for which hadrons are the relevant degrees of freedom.

We have found that for star matter a mixed hadron-quark phase precedes the pure quark matter, in agreement with previous studies. Also found are sudden changes in the compressibility at the extreme points of this mixed phase, as well as abrupt changes in the density rate of growth of the vector isovector meson and in the lepton concentration.

The effects of the finite baryon volume correlations on the neutron star structure have been examined, we have found that the maximum star mass is enhanced by both increasing
$B$ and introducing excluded volume corrections. The CC approach leads to a small increase of the star radius at the maximum mass, as compared to the respective $\mathrm{NC}$ case.

We have demonstrated that the $\mathrm{CC}$ treatment enlarges the range of applicability of the QMC model, which can be extended to reach the pure quark matter phase. In effect, in our approach this transition takes place at baryonic densities where excluded volume corrections are significative, and they should be included to describe properly the structure of massive systems, such as neutron stars.

Our results are based on a schematic model, however it seems to include the main ingredients for a reliable qualitative description of the high density regime of matter. Further refinements can be introduced to give more elaborate predictions, such as improved treatment of the quark matter phase and the consideration of exotic multiquark states, which will be the object of future studies.

\section{ACKNOWLEDGMENT}

This work was partially supported by CONICET, Argentina.
[1] H. Hatsuda and T. Kunihiro, Phys. Rep. 247, 221 (1994).

[2] G. E. Brown and M. Rho, Phys. Rep. 269, 333 (1996).

[3] J. Kapusta, Phys. Rev. D 23, 2444 (1981).

[4] A. Rakhimov, M. M. Musakhanov, F. C. Khanna, and U. Yakhshiev, Phys. Rev. C 58, 1738 (1998).

[5] P. A. M. Guichon, Phys. Lett. B 200, 235 (1988).

[6] K. Saito and A. W. Thomas, Phys. Lett. B 327, 9 (1994); Phys. Rev. C 51, 2757 (1995); K. Tsushima, K. Saito, and A. W. Thomas, Phys. Lett. B 411, 9 (1997); K. Tsushima, K. Saito, J. Haidenbauer, and A. W. Thomas, Nucl. Phys. A630, 691 (1998).

[7] K. Saito, K. Tsushima, and A. W. Thomas, nucl-th/9901084.

[8] S. Pal, M. Hanauske, I. Zakout, H. Stöcker, and W. Greiner, Phys. Rev. C 60, 015802 (1999).

[9] P. K. Panda, R. Sahu, and C. Das, Phys. Rev. C 60, 038801 (1999).

[10] R. Aguirre and A. L. De Paoli, nucl-th/9907087.

[11] S. Kagiyama, A. Nakamura, and T. Omodaka, Z. Phys. C 53, 163 (1992); 56, 557 (1992).

[12] S. Kagiyama, A. Minaka, and A. Nakamura, Prog. Theor. Phys. 89, 1227 (1993).

[13] D. H. Rischke, M. I. Gorenstein, H. Stöcker, and W. Greiner, Z. Phys. C 51, 485 (1991).

[14] J. Cleymans and H. Satz, Z. Phys. C 57, 135 (1993); J. Cleymans, M. I. Gorenstein, J. Stålnacke, and E. Suhonen, Phys. Scr. 48, 277 (1993); H. Kouno, K. Koide, T. Mitsumori, N.
Noda, A. Hasegawa, and M. Nakano, Prog. Theor. Phys. 96, 191 (1996); G. D. Yen, M. I. Gorenstein, W. Greiner, and S. N. Yang, Phys. Rev. C 56, 2210 (1997); M. I. Gorenstein, H. Stöcker, G. D. Yen, S. N. Yang, W. Greiner, J. Phys. G 24, 1777 (1998).

[15] C. P. Singh, B. K. Patra, and K. K. Singh, Phys. Lett. B 387, 680 (1996).

[16] J. D. Walecka, Ann Phys. 83, 491 (1974); J. Cleymans, Phys. Lett. 59B, 109 (1975).

[17] B. D. Serot and J. D. Walecka, Adv. Nucl. Phys. 16, 1 (1986); Int. J. Mod. Phys. E 6, 515 (1997).

[18] M. I. Gorenstein, A. P. Kostyuk, and Ya. D. Krivenko, J. Phys. G 25, L75 (1999).

[19] R. Knorren, M. Prakash, and P. J. Ellis, Phys. Rev. C 52, 3470 (1995); J. Schaffner and I. N. Mishustin, ibid. 53, 1416 (1996); K. Schertler, C. Greiner, J. Schaffner-Bielich, and M. H. Thoma, Nucl. Phys. A677, 463 (2000); N. K. Glendenning, Phys. Rep. 342, 393 (2001); J. Schaffner-Bielich, M. Hanauske, H. Stöcker, and W. Greiner, Phys. Rev. Lett. 89, 171101 (2002).

[20] N. K. Glendenning, Phys. Rev. D 46, 1274 (1992).

[21] G. Baym, C. Pethick, and P. Sutherland, Astrophys. J. 170, 299 (1971).

[22] J. W. Negele and D. Vautherin, Nucl. Phys. A207, 298 (1973).

[23] J. B. Hartle, Astrophys. J. 150, 1005 (1967); A. Akmal, V. R. Pandharipande, and D. G. Ravenhall, Phys. Rev. C 58, 1804 (1998). 